\title{
Optimal Contract Design for Ancillary Services in Vehicle-to-Grid Networks
}

\author{
Yang Gao*, Yan Chen*, Chih-Yu Wang ${ }^{\dagger}$, and K. J. Ray Liu* \\ * Department of Electrical and Computer Engineering, University of Maryland, College Park, MD 20742, USA \\ ${ }^{\dagger}$ Graduate Institute of Communication Engineering, National Taiwan University, Taipei, Taiwan \\ E-mail: *\{yanggao, yan, kjrliu $\} @ u m d . e d u ; ~ †$ tomkywang@gmail.com
}

\begin{abstract}
With the foreseeable large scale deployment of electric vehicles (EVs) and the development of vehicle-to-grid (V2G) technologies, it is possible to provide ancillary services to the power grid in a cost efficient way, i.e., through the bidirectional power flow of EVs. A key issue in such kind of schemes is how to stimulate a large number of EVs to act coordinately to achieve the service request. This is challenging since EVs are self-interested and generally have different preferences toward charging and discharging based on their own constraints. In this paper, we propose a contract-based mechanism to tackle this challenge. Through the design of an optimal contract, the aggregator can provide incentives for EVs to participate in ancillary services to power grid, match the aggregated energy rate with the service request and maximize its own utility. Simulation results are shown to verify the effectiveness of the proposed contract-based mechanism.
\end{abstract}

\section{INTRODUCTION}

Due to the need of reducing oil dependence and improving energy efficiency, many countries including United States have set up plans to support electric vehicles (EVs) [1]. It is predicated in [1] that by $2020,25 \%$ of newly purchased lightduty vehicles should be grid-enabled EVs. Such a widespread adoption of EVs, together with the development of vehicle-togrid (V2G) technologies [2], will open new opportunities for the power grid: using EVs' batteries as distributed electricity storage, it is possible to provide ancillary services to power grid, such as spinning reserve and regulation, in a cost efficient way. In current power grid, these services are accomplished primarily by turning large generators on and off or ramping them up and down, which are very costly, e.g., accounting for $5-10 \%$ of electric cost in the US [3]. Alternatively, in V2G networks, these services can be done by charging (or discharging) EVs' batteries when the generation is greater (or less) than the load in power grid. Due to the limited capacity of an individual $\mathrm{EV}$, the aggregator is introduced to coordinate a large group of EVs in providing ancillary services to the power grid [4].

Recently, a growing body of literature has investigated different charging control schemes for the aggregator. In [5], $\mathrm{Xu}$ and Wong proposed a coordinated charging control method that uses approximate dynamic programming to minimize the charging cost and reduce the power losses. Wu et al. proposed algorithms that help the aggregator to determine the purchase of energy in the day-ahead market and to distribute the purchased energy to EVs [6]. Among these works, many of them have studied the use of EVs for ancillary services. Frequency regulation has been considered in [7], where an optimal centralized control strategy was proposed. In [8], Sortomme et al. demonstrated an optimal energy and ancillary service scheduling strategy that maximizes profits to the aggregator.

Most of existing works assume implicitly that EVs are obedient to aggregator's policies. Nevertheless, with the development of smart grid technologies [9], EVs are capable of making intelligent decisions to maximize their own utilities. Therefore, a key issue in enabling ancillary services in V2G networks is to provide incentives for EVs to participate. In [10], Wu et al. modeled the interaction between the aggregator and EVs as a game and proposed a flat price scheme to accomplish the ancillary service request at the equilibrium. However, one major drawback is that they assume a homogeneous setting without taking into account different preferences of EVs. In practice, since EVs generally have different constraints, such as arrival time, departure time, initial battery level and target battery level, they will have different preferences toward charging/discharging at different time. Moreover, such preferences are generally unknown to the aggregator, which results in an information asymmetry between the aggregator and EVs.

To tackle this challenge, we first model EV's preference as a willingness to pay (WTP) parameter [11] that reflects the private and subjective valuation of each EV towards charging/discharging its battery. Then, based on this heterogeneous model, we solve the incentive issue in EV-assisted ancillary services using contract theory, which studies, in the presence of asymmetric information, how the principal (the aggregator) delegates an action (charging/discharing at a certain rate) to intelligent and selfish agents (EVs) through a take-it-or-leave-it offer of a contract [12]. With the design of an optimal contract, the aggregator not only can stimulate self-interested EVs to act coordinately to provide ancillary services to power grid, but also maximizes its own utility.

The rest of the paper is organized as follows. In Section II, we introduce the system model and problem formulation. Then, the optimal contract design is discussed in Section III. In Section IV, we develop an algorithm that finds discrete optimal contracts efficiently. Finally, we show simulation results in Section V and draw conclusions in Section VI. 


\section{System Model AND PROBlem Formulation}

\section{A. V2G System Model}

Consider a V2G network where a group of $N$ EVs are interested in providing ancillary services to the power grid by charging or discharging their batteries. One aggregator is responsible for coordinating the participating EVs. However, the aggregator has no direct control over the charging/discharging behaviors of EVs, who are assumed to be self-interested and will act selfishly to maximize their own utilities.

We divide the daily operation of the power grid into multiple time slots, each of which corresponds to one service period. At each time slot, the grid sends a service request to the aggregator indicating the aggregated energy rate needed from the aggregator in order to accomplish the ancillary service. Denote by $\Delta$ the service request sent to the aggregator. We study the nontrivial case that $\Delta \neq 0$ in this paper. If $\Delta>0$, the aggregator needs to consume power. If $\Delta<0$, the aggregator needs to inject power into the power grid. Such a service request is accomplished by the aggregator through coordinating the $N$ associated EVs to charge or discharge their batteries. Moreover, similar to [10], we assume that the aggregator is equipped with a set of backup batteries to assure reaching the service request.

We consider a heterogeneous setting where EVs have different preferences toward charging/discharging at each time slot, which are modeled by a WTP parameter $\theta$ [11]. Let $r$ denote the charging/discharging rate of an EV and $p$ denote the price paid to the aggregator. Then the utility function of the EV with WTP parameter $\theta$ can be written as

$$
u_{\theta}(r, p)= \begin{cases}\theta r-p, & \text { if } r \geq 0, \\ (\mathcal{C}+\theta) r-p, & \text { otherwise }\end{cases}
$$

where $\mathcal{C}>0$ is the unit cost associated with discharging.

Note that both $r$ and $p$ can take either positive or negative values. In particular, $r>0$ means the EV charges its battery at current time slot while $r<0$ means discharging. Based on the utility function, the WTP parameter can be interpreted as the unit gain that an EV can receive by charing or discharging its battery. In this paper, we model the WTP parameter $\theta \in$ $\Theta=[\underline{\theta}, \bar{\theta}]$ as a random variable, with a cumulative distribution function (CDF) $F(\theta)$ and a probability density function (PDF) $f(\theta)$ on $\Theta$. We assume that $-\mathcal{C}<\underline{\theta}<0$ and $\bar{\theta}>0$. The sign of $\theta$ indicates whether the EV tends to charge or discharge: when $\theta>0$, the EV prefers to charge; when $\theta<0$ the EV prefers to discharge and when $\theta=0$ the $\mathrm{EV}$ wants to remain idle. Moreover, the larger $|\theta|$ is, the more an EV wants to charge or discharge its battery, respectively. As an independent decision-maker, each EV will act to maximize its own utility function in (1) without considering whether the aggregated load matches the service request or not. Therefore, an inherent conflict exists in terms of objectives between the aggregator and EVs.

We further assume that the WTP parameter is the private information of each EV, which we call as the EV's type. In other words, instead of knowing the specific value of each EV's type, the aggregator only has the knowledge of $f(\theta)$ and
$F(\theta)$. As a result, there exists an information asymmetry between the aggregator and EVs, which makes the coordination at the aggregator even harder.

\section{B. Contract-Theoretic Formulation}

To resolve the conflicting objectives between the aggregator and EVs in the presence of asymmetric information, we propose to use a contract-theoretic approach. Through an optimal design of contract, the aggregator can not only stimulate selfinterested EVs to act coordinately to accomplish the service request but also maximize its own utility. In contract theory, a contract is a collection of contract items. Particularly, in our case, each contract item corresponds to a pair $(r, p)$, which specifies the EV's charging/discharging rate and the resulted payment to the aggregator. At each time slot, the aggregator will publish the contract to all participating EVs. Then each EV will choose one contract item that maximizes its utility defined in (1). According to the revelation principle [13], it is sufficient to consider the class of contracts that ensure each EV to truthfully choose the contract item designed for its type. Therefore, we can design our contract as a pair of functions as $\phi=\{(r(\theta), p(\theta)), \theta \in \Theta\}$. Throughout this paper, we assume both the rate function $r(\theta)$ and the price function $p(\theta)$ are continuously differentiable, while our analysis results can be extended to the piecewise continuously differentiable case. To be a feasible contract, $\phi$ needs to satisfy the incentive compatibility (IC) constraint and the individual rationality (IR) constraint, which we define as follows.

Definition 1 (Incentive Compatibility). A contract $\phi=$ $\{(r(\theta), p(\theta)), \theta \in \Theta\}$ satisfies the incentive compatibility constraint if it is the best response of each $\theta$-type EV to choose the contract item for type $\theta$, i.e.,

$$
u_{\theta}(r(\theta), p(\theta)) \geq u_{\theta}(r(\tilde{\theta}), p(\tilde{\theta})), \quad \forall \theta, \tilde{\theta} \in \Theta .
$$

Definition 2 (Individual Rationality). A contract $\phi=$ $\{(r(\theta), p(\theta)), \theta \in \Theta\}$ satisfies the individual rationality constraint if each $\theta$-type $E V$ receives a non-negative utility by accepting the contract item for type $\theta$, i.e.,

$$
u_{\theta}(r(\theta), p(\theta)) \geq 0, \quad \forall \theta \in \Theta .
$$

A contract that satisfies the IR constraint will provide nonnegative utilities to EVs of any types, and therefore ensures the participation of self-interested EVs.

In addition to the IC and IR constraints, the aggregator will design the contract such that the expected aggregated energy rate of all EVs meets the service request, i.e.,

$$
N \int_{\underline{\theta}}^{\bar{\theta}} r(\theta) f(\theta) d \theta=\Delta .
$$

Moreover, we place the following two constraints on the design of contracts

$$
r(\theta) \Delta \geq 0, \quad \forall \theta \in \Theta
$$

and

$$
r(0)=0 \text {. }
$$

The first constraint indicates that charging and discharging should not be performed simultaneously. Otherwise EVs cancel out each other's efforts in terms of providing ancillary 
services to the power grid. The second constraint indicates that we should not involve EVs that choose to remain idle into the ancillary service.

The rate function must also satisfy

$$
r_{\text {min }} \leq r(\theta) \leq r_{\max }, \quad \forall \theta \in \Theta,
$$

where $r_{\max }>0$ and $r_{\min }<0$ are the maximum rates of EVs to charge and discharge, respectively.

Denote by $\Phi$ the set of contracts that satisfy all constraints in (2)-(7). Assume $N$ is sufficiently large that $\Phi$ is not empty. Among all contracts in $\Phi$, the aggregator will choose the optimal one, which maximizes its utility as

$$
\phi^{*}=\max _{\phi \in \Phi} \int_{\underline{\theta}}^{\bar{\theta}}\left[p(\theta)-\lambda \dot{r}^{2}(\theta)\right] f(\theta) d \theta,
$$

where $\dot{r}(\theta)=\frac{d}{d \theta} r(\theta)$ is the derivative of $r(\theta)$.

The utility of an aggregator is defined as the total profit plus a regularization term, whose weight is controlled by a parameter $\lambda>0$. The regularization term is introduced to prevent the charging/discharging rate from varying too quickly among neighboring types, which may cause instability due to estimation errors of types. From another point of view, we know in a simple scheme where EVs are assumed to be homogeneous, the aggregator offers a flat charging/discharging rate to all $\mathrm{EVs}$, i.e. with $\dot{r}(\theta)=0$. Therefore, through the introduction of the regularization term, the aggregator can choose how far it would like to deviate from the simple scheme by adjusting $\lambda$.

The proposed contract-based mechanism in one time slot can be summarized in the following four steps.

1) The aggregator receives the service request from the power grid and calculates the optimal contract $\phi^{*}$

2) The aggregator broadcasts $\phi^{*}$ to all EVs

3) After receiving $\phi^{*}$, each EV selects one contract item that maximizes its utility and informs the aggregator its decision

4) The aggregator coordinates the ancillary service and records EVs' payments given the selected contract items

\section{Optimal Contract Design}

To find the optimal contract, we need to solve the optimization problem defined in (8), which is challenging because it optimizes over a class of functions specified by some complicated constraints. In this section, we first simplify the optimization problem to a certain extent by finding equivalent conditions to the IC and IR constraints. Then, by using the optimal control theory, we characterize the necessary and sufficient conditions of the optimal contract. In the rest of this paper, we assume without loss of generality that $\Delta>0$ in our analysis. The case with $\Delta<0$ can be analyzed similarly.

We show in the following two propositions that the IC and IR constraints can be simplified in our problem settings.

Proposition 1. Suppose $\Delta>0$ and a contract $\phi=$ $\{(r(\theta), p(\theta)), \theta \in \Theta\}$ satisfies the efficiency constraint defined in (5). Then $\phi$ satisfies the IC constraint if and only if, $\forall \theta \in \Theta$,

$$
\dot{r}(\theta) \geq 0
$$

and

$$
\theta \dot{r}(\theta)-\dot{p}(\theta)=0 .
$$

Proof: From $\Delta>0$ and (5), we have $r(\theta) \geq 0, \forall \theta \in \Theta$, which implies $u_{\theta}(r(\theta), p(\theta))=\theta r(\theta)-p(\theta)$.

To prove Proposition 1, we first show that the two conditions in (9) and (10) are necessary conditions for the IC constraint. From Definition 1, we have $\forall \theta, \tilde{\theta} \in \Theta$,

and

$$
\theta r(\theta)-p(\theta) \geq \theta r(\tilde{\theta})-p(\tilde{\theta}),
$$

$$
\tilde{\theta} r(\tilde{\theta})-p(\tilde{\theta}) \geq \tilde{\theta} r(\theta)-p(\theta) .
$$

Adding the above two inequalities, we have

$$
(\theta-\tilde{\theta})(r(\theta)-r(\tilde{\theta})) \geq 0, \forall \theta, \tilde{\theta} \in \Theta .
$$

Therefore, we can conclude that $\dot{r}(\theta) \geq 0, \forall \theta \in \Theta$.

Moreover, let

$$
g_{\theta}(\tilde{\theta}) \triangleq \theta r(\tilde{\theta})-p(\tilde{\theta}) .
$$

Then the IC constraint implies that

$$
\theta \in \arg \max _{\tilde{\theta} \in \Theta} g_{\theta}(\tilde{\theta}), \quad \forall \theta \in \Theta .
$$

Since $g_{\theta}(\tilde{\theta})$ is continuously differentiable, from the first-order optimality condition [14], we have

$$
\left.\frac{\partial g_{\theta}(\tilde{\theta})}{\partial \tilde{\theta}}\right|_{\tilde{\theta}=\theta}=\theta \frac{d}{d \theta} r(\theta)-\frac{d}{d \theta} p(\theta)=0, \quad \forall \theta \in(\underline{\theta}, \bar{\theta}) .
$$

Then, by continuity of $\dot{r}(\theta)$ and $\dot{p}(\theta)$, we can establish (10).

Next, we prove conditions in (9) and (10) are also sufficient conditions for the IC constraint. We have $\forall \theta, \tilde{\theta} \in \Theta$,

$$
\begin{aligned}
p(\theta)-p(\tilde{\theta}) & =\int_{\tilde{\theta}}^{\theta} \dot{p}(\tau) d \tau=\int_{\tilde{\theta}}^{\theta} \tau \dot{r}(\tau) d \tau \\
& =\theta r(\theta)-\tilde{\theta} r(\tilde{\theta})-\int_{\tilde{\theta}}^{\theta} r(\tau) d \tau,
\end{aligned}
$$

where the second equality follows from (10) and the last equality is obtained through integration by parts.

After some manipulations, we have

$$
\begin{aligned}
\theta r(\theta)-p(\theta) & =\operatorname{\theta r}(\tilde{\theta})-p(\tilde{\theta})+\int_{\tilde{\theta}}^{\theta}[r(\tau)-r(\tilde{\theta})] d \tau \\
& \geq \operatorname{\theta r}(\tilde{\theta})-p(\tilde{\theta}), \quad \forall \theta, \tilde{\theta} \in \Theta,
\end{aligned}
$$

where the inequality follows from (9).

Proposition 2. Suppose $\Delta>0$ and a contract $\phi=$ $\{(r(\theta), p(\theta)), \theta \in \Theta\}$ satisfies the IC constraint and the efficiency constraint defined in (5). Then, $\phi$ satisfies the IR constraint if and only if

$$
\underline{\theta} r(\underline{\theta})-p(\underline{\theta}) \geq 0 .
$$

Proof: Let $U(\theta) \triangleq \theta r(\theta)-p(\theta)$. Since $\phi$ satisfies the IC constraint, according to (10), we have

$$
\dot{U}(\theta)=\frac{d}{d \theta} U(\theta)=r(\theta)+\theta \dot{r}(\theta)-\dot{p}(\theta)=r(\theta), \forall \theta \in \Theta .
$$

Since $\Delta>0$, then (5) implies that $r(\theta) \geq 0, \forall \theta \in \Theta$. Therefore, we have

$$
\underline{\theta} \in \arg \min _{\theta \in \Theta} U(\theta) .
$$

The IR constraint in (3) is thus equivalent to $U(\underline{\theta}) \geq 0$. 
Based on the definition of $U(\theta)$, we can rewrite the objective function in (8) as

$$
G(\phi) \triangleq \int_{\underline{\theta}}^{\bar{\theta}}\left[\theta r(\theta)-\lambda \dot{r}^{2}(\theta)\right] f(\theta) d \theta-\int_{\underline{\theta}}^{\bar{\theta}} U(\theta) f(\theta) d \theta .
$$

The last term can be expressed in terms of $r(\theta)$ as

$$
\begin{aligned}
\int_{\underline{\theta}}^{\bar{\theta}} U(\theta) f(\theta) d \theta & =\int_{\underline{\theta}}^{\bar{\theta}} f(\theta) \int_{\underline{\theta}}^{\theta} \dot{U}(\tau) d \tau d \theta+U(\underline{\theta}) \\
& =\int_{\underline{\theta}}^{\bar{\theta}} f(\theta) \int_{\underline{\theta}}^{\theta} r(\tau) d \tau d \theta+U(\underline{\theta}) \\
& =\int_{\underline{\theta}}^{\bar{\theta}} r(\theta) d \theta-\int_{\underline{\theta}}^{\bar{\theta}} r(\theta) F(\theta) d \theta+U(\underline{\theta}),
\end{aligned}
$$

where the second equality follows (20) and the last equality is obtained through integration by parts.

Therefore, we have

$G(\phi)=\int_{\underline{\theta}}^{\bar{\theta}} r(\theta)[\theta f(\theta)+F(\theta)-1]-\lambda \dot{r}^{2}(\theta) f(\theta) d \theta-U(\underline{\theta})$.

We can simplify $G(\phi)$ by the following two observations. First, since $r(\theta) \geq 0, \forall \theta \in \Theta$, we can conclude from (6) and (9) that $r(\theta)=0$ and $\dot{r}(\theta)=0$ for $\theta \in[\theta, 0]$. Second, we can maximize $G(\phi)$ while satisfying the IR constraint by setting $U(\underline{\theta})=0$, i.e. $p(\underline{\theta})=0$.

Therefore, the optimal contract design problem in (8) can be simplified to a constrained optimization problem with respect to the rate function $r(\theta)$ over $[0, \bar{\theta}]$ as

$$
\begin{aligned}
\max _{r(\theta)} & \int_{0}^{\bar{\theta}} r(\theta)[\theta f(\theta)+F(\theta)-1]-\lambda \dot{r}^{2}(\theta) f(\theta) d \theta \\
\text { subject to } & \int_{0}^{\bar{\theta}} r(\theta) f(\theta) d \theta=\frac{\Delta}{N}, \\
& r(0)=0, \\
& \dot{r}(\theta) \geq 0 \text { and } r(\theta) \leq r_{\max }, \quad \forall \theta \in[0, \bar{\theta}] .
\end{aligned}
$$

Once we have found the optimal rate function $r^{*}(\theta)$, we can determine the optimal pricing function $p^{*}(\theta)$ as

$$
p^{*}(\theta)=\int_{\underline{\theta}}^{\theta} \tau r^{*}(\tau) d \tau .
$$

To solve (22), we employ the Pontryagin's maximum principle [15] in optimal control theory to obtain a set of necessary conditions for the optimal rate function $r^{*}(\theta)$. Our results are summarized in the following theorem.

Theorem 1. If $r^{*}(\theta)$ is a solution to (22), then there must exist $x_{1}^{*}(\theta), x_{2}^{*}(\theta), x_{3}^{*}(\theta)$ and $y_{1}^{*}(\theta)$, which are defined on $[0, \bar{\theta}]$ and are solutions to the following differential equations, such that $r^{*}(\theta)=x_{1}^{*}(\theta)$.

$$
\begin{aligned}
& \dot{x}_{1}^{*}(\theta)=\frac{y_{1}^{*}(\theta)}{2 \lambda f(\theta)} \mathbf{1}\left(y_{1}^{*}(\theta) \geq 0\right), \\
& \dot{x}_{2}^{*}(\theta)=f(\theta) x_{1}^{*}(\theta), \\
& \dot{x}_{3}^{*}(\theta)=\left[r_{\max }-x_{1}^{*}(\theta)\right]^{2} \mathbf{1}\left(x_{1}^{*}(\theta)>r_{\max }\right), \\
& \dot{y}_{1}^{*}(\theta)=1-F(\theta)-\left(\alpha_{1}+\theta\right) f(\theta)-2 \alpha_{2}\left[x_{1}^{*}(\theta)-r_{\max }\right] \mathbf{1}\left(x_{1}^{*}(\theta)>r_{\max }\right),
\end{aligned}
$$

where $\alpha_{1}$ and $\alpha_{2}$ are two constants to be determined. The associated boundary conditions are $x_{1}^{*}(0)=x_{2}^{*}(0)=x_{3}^{*}(0)=$ $x_{3}^{*}(\bar{\theta})=y_{1}^{*}(\bar{\theta})=0$ and $x_{2}^{*}(\theta)=\Delta / N$.

Proof: The optimization problem in (22) can be viewed as an optimal control problem if we treat $r(\theta)$ as the state variable, $\dot{r}(\theta)$ as the control and $\theta$ as the time variable. Let $x_{1}(\theta)=r(\theta)$ and $u(\theta)=\dot{r}(\theta)$. To re-write (22) in a standard form, we need to get rid of the isoperimetric constraint $\int_{0}^{\bar{\theta}} r(\theta) f(\theta) d \theta=\frac{\Delta}{N}$ and the state constraint $r(\theta) \leq r_{\max }$. Towards this end, we define two new state variables $x_{2}(\theta)$ and $x_{3}(\theta)$, such that

and

$$
\dot{x}_{2}(\theta)=f(\theta) x_{1}(\theta)
$$

$$
\dot{x}_{3}(\theta)=\left[r_{\max }-x_{1}(\theta)\right]^{2} \mathbf{1}\left(x_{1}(\theta)>r_{\max }\right) .
$$

Then, by specifying the boundary conditions $x_{2}(0)=0$ and $x_{2}(\bar{\theta})=\Delta / N$, we guarantee that $\int_{0}^{\bar{\theta}} r(\theta) f(\theta) d \theta=\frac{\Delta}{N}$. Similarly, by setting $x_{3}(0)=x_{3}(\bar{\theta})=0$, we ensure that $r(\theta) \leq r_{\text {max }}$.

Let $\mathbf{x}(\theta)=\left[x_{1}(\theta), x_{2}(\theta), x_{3}(\theta)\right]^{T}$. We can write the Hamiltonian [15] of the problem as

$$
\begin{aligned}
\mathcal{H}(\mathbf{x}(\theta), u(\theta), \mathbf{y}(\theta), \theta) & \triangleq x_{1}(\theta)[\theta f(\theta)+F(\theta)-1]-\lambda u^{2}(\theta) f(\theta) \\
& +y_{1}(\theta) u(\theta)+y_{2}(\theta) f(\theta) x_{1}(\theta) \\
& +y_{3}(\theta)\left[r_{\max }-x_{1}(\theta)\right]^{2} \mathbf{1}\left(x_{1}(\theta)>r_{\max }\right),
\end{aligned}
$$

where $\mathbf{y}(\theta)=\left[y_{1}(\theta), y_{2}(\theta), y_{3}(\theta)\right]^{T}$ is the vector of Lagrange multipliers.

Then, according to the Pontryagin's maximum principle [15], we can write the necessary conditions of the optimal control and states in terms of the Hamiltonian as

$$
\left\{\begin{array}{l}
\mathcal{H}\left(\mathbf{x}^{*}(\theta), u^{*}(\theta), \mathbf{y}^{*}(\theta), \theta\right) \geq \mathcal{H}\left(\mathbf{x}^{*}(\theta), u(\theta), \mathbf{y}^{*}(\theta), \theta\right), \forall u(\theta) \geq 0, \\
\dot{\mathbf{x}}^{*}(\theta)=\frac{\partial \mathcal{H}}{\partial \mathbf{y}}\left(\mathbf{x}^{*}(\theta), u^{*}(\theta), \mathbf{y}^{*}(\theta), \theta\right), \\
\dot{\mathbf{y}}^{*}(\theta)=-\frac{\partial \mathcal{H}}{\partial \mathbf{x}}\left(\mathbf{x}^{*}(\theta), u^{*}(\theta), \mathbf{y}^{*}(\theta), \theta\right),
\end{array}\right.
$$

with the boundary condition $y_{1}^{*}(\bar{\theta})=0$.

Since the Hamiltonian does not depend on $x_{2}(\theta)$ and $x_{3}(\theta)$, we have from (26) that $\dot{y}_{2}(\theta)=\dot{y}_{3}(\theta)=0$. This implies that the Lagrange multipliers $y_{2}(\theta)$ and $y_{3}(\theta)$ are constants, which we denote by $\alpha_{1}$ and $\alpha_{2}$, respectively. From (26) and the boundary conditions, we can derive the necessary conditions in Theorem 1.

In Theorem 1, we have characterized the necessary conditions of $r^{*}(\theta)$ in terms of a set of differential equations with split boundary conditions, which is also called as a two-point boundary-value problem [15]. In general, this twopoint boundary-value problem can be solved numerically by some iterative techniques [15]. Instead of solving the above necessary conditions numerically, we show in the following theorem that when the maximum rate $r_{\max }$ is sufficiently large, we can obtain an analytical expression for $r^{*}(\theta)$ that is also sufficient.

Theorem 2. If $r_{\max }$ is sufficiently large, then the solution of (22) is given by

$$
r^{*}(\theta)=\int_{0}^{\theta} \frac{y_{1}^{*}(\tau)}{2 \lambda f(\tau)} \mathbf{1}\left(y_{1}^{*}(\tau) \geq 0\right) d \tau,
$$


where

$$
y_{1}^{*}(\theta)=\int_{\bar{\theta}}^{\theta}[1-F(\tau)-(\alpha+\tau) f(\tau)] d \tau
$$

and the constant $\alpha$ can be determined by

$$
\int_{0}^{\bar{\theta}} \frac{1-F(\tau)}{f(\tau)} y_{1}^{*}(\tau) \mathbf{1}\left(y_{1}^{*}(\tau) \geq 0\right) d \tau=\frac{2 \lambda \Delta}{N} .
$$

Proof: When $r_{\max }$ is sufficiently large, the state constraint $r(\theta) \leq r_{\max }$ will not be binding. Therefore, we no longer need the state variable $x_{3}(\theta)$ as in the proof of Theorem 1. Then, applying the Pontryagin's maximum principle and after some manipulations, we can derive the conditions in Theorem 2 as necessary conditions for $r^{*}(\theta)$.

Next, we show that the conditions we obtained are also sufficient for the optimal rate function. As in the proof of Theorem 1, we can write the Hamiltonian as

$$
\begin{aligned}
\mathcal{H}(\mathbf{x}(\theta), u(\theta), \mathbf{y}(\theta), \theta) & \triangleq x_{1}(\theta)[\theta f(\theta)+F(\theta)-1]-\lambda u^{2}(\theta) f(\theta) \\
& +y_{1}(\theta) u(\theta)+y_{2}(\theta) f(\theta) x_{1}(\theta) .
\end{aligned}
$$

Since

$$
\begin{aligned}
\mathcal{H}^{*}(\mathbf{x}, \mathbf{y}, \theta) & \triangleq \max _{u \geq 0} \mathcal{H}(\mathbf{x}, u, \mathbf{y}, \theta) \\
& =\left[\theta f(\theta)+F(\theta)-1+y_{2} f(\theta)\right] x_{1}+y_{1} u^{*}-\lambda f(\theta) u^{*}
\end{aligned}
$$

is concave in $\mathbf{x}$, the conditions obtained from the Pontryagin's maximum principle are also sufficient [16].

Therefore, the conditions in Theorem 2 are complete characterizations of $r^{*}(\theta)$.

Example 1. Consider an illustrating example, where $\theta$ is uniform within $[-1,1]$, i.e., $f(\theta)=1 / 2$ and $F(\theta)=(1+\theta) / 2$. Then, applying Theorem 2, we can obtain the optimal rate function on $[0, \bar{\theta}]$ as follows.

- If $\frac{48 \lambda \Delta}{N} \geq 1$,

$$
r^{*}(\theta)=\frac{1}{2 \lambda}\left[-\frac{\theta^{3}}{3}+\frac{1-\alpha}{2} \theta^{2}+\alpha \theta\right],
$$

where $\alpha=\frac{12 \lambda \Delta}{N}-\frac{1}{4}$.

$$
\begin{aligned}
& \text { - If } \frac{48 \lambda \Delta}{N}<1, \\
& r^{*}(\theta)=\frac{1}{2 \lambda}\left[-\frac{\theta^{3}}{3}+\frac{1-\alpha}{2} \theta^{2}+\alpha \theta+\frac{\alpha^{3}+3 \alpha^{2}}{6}\right] \mathbf{1}(\theta \geq-\alpha),
\end{aligned}
$$

where $\alpha$ is the unique solution in the interval $(-1,0)$ to

$$
\alpha^{4}+4 \alpha^{3}+6 \alpha^{2}+4 \alpha+1=\frac{48 \lambda \Delta}{N} .
$$

\section{Discrete Optimal Contract Design}

In previous section, we provide necessary and sufficient conditions for the optimal contract. Nevertheless, these conditions are hard to evaluate when the EV's type follows a complicated distribution or when the maximum rate $r_{\max }$ is relatively small. To overcome these difficulties, we present in this section a numerical algorithm that finds a discrete optimal contract by approximating the optimization problem in (22) with a discrete problem. Towards this end, let

$$
\left\{\begin{array}{l}
r_{k} \triangleq r(k \delta), k=0,1, \ldots, K \\
u_{k} \triangleq \dot{r}(k \delta), k=0,1, \ldots, K
\end{array}\right.
$$

where $\delta=\frac{\bar{\theta}}{K+1}$. Since $r(k \delta+\delta) \approx r(k \delta)+\dot{r}(k \delta) \delta$, we can approximate $r_{k}$ by $r_{k}=\delta \sum_{i=0}^{k-1} u_{i}$. Note that we have $r_{0}=0$ as indicated by the constraint of (22).

Then, by approximating the integrals with summations and after some manipulations, we derive a discrete approximation of (22) as

$$
\begin{array}{ll}
\min _{u_{0}, u_{1}, \ldots, u_{K-1}} & \sum_{k=0}^{K-1}\left(B_{k} u_{k}^{2}-A_{k} u_{k}\right), \\
\text { subject to } & \sum_{k=0}^{K-1} D_{k} u_{k}-\frac{\Delta}{N}=0, \\
& -u_{k} \leq 0, \quad k=0,1, \ldots, K-1, \\
& \delta \sum_{k=0}^{K-1} u_{k}-r_{\max } \leq 0,
\end{array}
$$

where $A_{k}=\delta \sum_{i=k+1}^{K}[i \delta f(i \delta)+F(i \delta)-1], B_{k}=\lambda f(k \delta)$ and $D_{k}=\delta^{2} \sum_{i=k+1}^{K} f(i \delta)$.

The (31) is a constrained quadratic programming problem that can be solved efficiently by the primal-dual inner-point method [14]. We skip the details of the algorithm due to page limitation. Denote the solution to (31) by $u_{1}^{*}, \ldots, u_{K-1}^{*}$. Then, after solving (31), we can design a discrete optimal contract $\phi^{d i s}=\left\{\left(r^{d i s}(\theta), p^{d i s}(\theta)\right), \theta \in \Theta\right\}$ as

$$
\left\{\begin{array}{l}
r^{\text {dis }}(\theta)=\sum_{k=1}^{K} \delta u_{k-1}^{*} \mathbf{1}(\theta \geq k \delta) \\
p^{\text {dis }}(\theta)=\sum_{k=1}^{K}(k-1) \delta^{2} u_{k-1}^{*} \mathbf{1}(\theta \geq k \delta) .
\end{array}\right.
$$

\section{Simulation Results}

In this section, we conducted numerical simulations to evaluate the proposed contract-based mechanism. A V2G system with $N=1000 \mathrm{EVs}$ is considered. We assume EV's WTP parameters are independent and identically distributed within $[-1,1]$ uniformly. The unit cost $\mathcal{C}$ is assumed to be 1.1. Moreover, we set $r_{\max }=7.68 \mathrm{~kW}$ and $r_{\min }=-7.68 \mathrm{~kW}$ based on the Level 2 charging standard in North America [10].

In the first simulation, we compare the discrete optimal rate function obtained by solving (31) with the theoretic results in Theorem 2. Simulation results for two cases, where $\Delta=$ $2(M W)$ and $\Delta=0.5(M W)$, are shown in Fig. 1. In both cases, we set $\lambda=0.02$. We can see that as $K$ increases, the discrete optimal rate functions are getting closer to theoretic results. Moreover, the two curves almost coincide when $K=$ 100 , which verifies that $\phi^{d i s}$ is a good approximation for $\phi^{*}$ with large $K \mathrm{~s}$.

Next, we evaluate the performance of the proposed contractbased mechanism under the scenario of frequency regulation. Simulation results are shown in Fig. 2. We assume $\Delta$ is updated every 1 minute by the power grid and its value follows a Gaussian distribution with zero mean and variance of $1\left(M W^{2}\right)$, as shown in Fig. 2(a). Moreover, $\lambda$ is set as 0.001 for the aggregator. We show in Fig. 2(b) the difference 


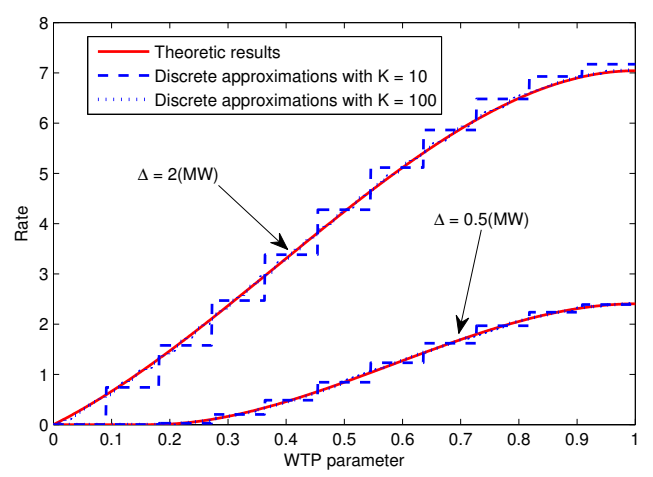

Fig. 1. The rate function versus the WTP parameter values.

between $\Delta$ and the aggregated energy rate of all EVs by using the proposed mechanism. We can see that with the proposed mechanism, the aggregator can achieve over $95 \%$ of the service request. The differences are not zeros due to the randomness of EV's WTP parameter.

We then compare the proposed mechanism with a flat price scheme [10] in terms of the total payment received by the aggregator. In [10], to achieve the service request, the aggregator randomly selects a certain number of EVs to charge/discharge their batteries at a fixed rate. The aggregator will pay each selected EV a base price $\omega$, which is the same for all selected EVs, and charge them penalty prices if the service request can not be reached. Therefore, to avoid penalties, the selected EVs will follow the aggregator's instructions if they can receive non-negative utilities. Otherwise they will simply choose to remain idle. Since the aggregator does not know each EV's preference, the base price should be large enough so that every selected EV will have the incentive to participate. In the simulation, we set the fixed charging/discharging rate as $r_{\max } / r_{\min }$ and the base price as $\omega=\max _{\theta \in \Theta} \theta r_{\max }=7.68$ when $\Delta>0$ and $\omega=\max _{\theta \in \Theta}(\theta+\mathcal{C}) r_{\min }=16.128$ when $\Delta<0$. The total payment received by the aggregator using the proposed mechanism, $P^{o p t}$, is shown in Fig. 2(c) and that using the flat price scheme, $P^{\text {flat }}$, is shown in Fig. 2(d). We also show the difference between $P^{o p t}$ and $P^{\text {flat }}$ in Fig. 2(e). From the simulation results, we can see that the optimal contract enables the aggregator to exploit different preferences of EVs and therefore to extract more profit while achieving the service request statistically. On the other hand, in the flat price scheme, the aggregator always has to overpay the EVs, which results in a loss of profit for the aggregator.

\section{Vi. Conclusions}

In this paper, we study the problem of stimulating selfinterested EVs in providing ancillary services to the power grid. A heterogeneous setting is considered where EVs have different preferences toward charging/discharging at different time. We propose a contract-based mechanism and characterize the necessary and sufficient conditions of the optimal contract. By using the optimal contract, aggregator can maximize its utility while coordinating EVs to satisfy the service request. We also develop a numerical algorithm to find discrete

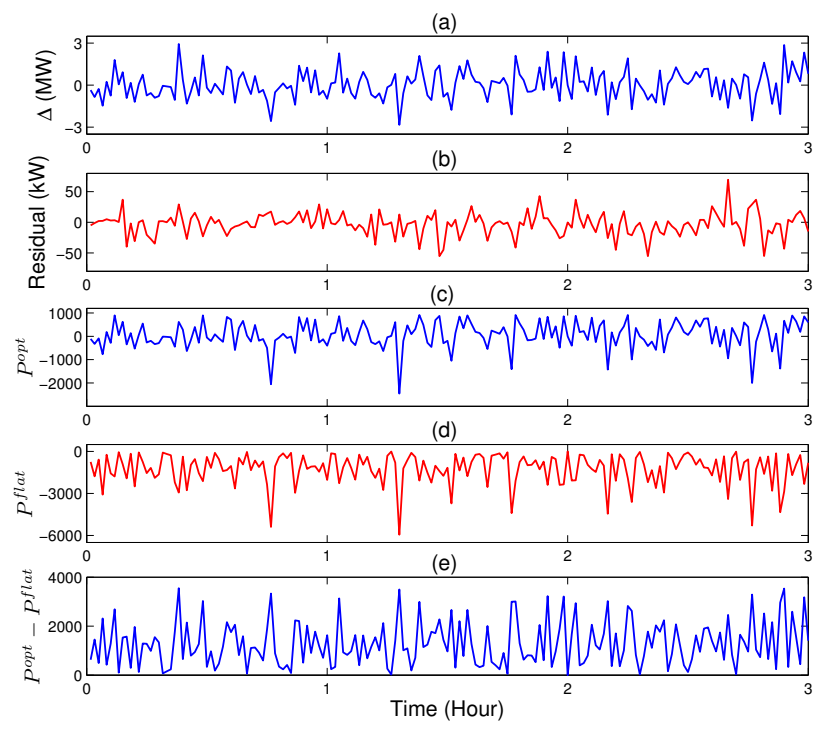

Fig. 2. Simulation results for frequency regulation: (a) The service request. (b) The difference between service request and the aggregated energy rate of all EVs. (c) The total payment received by the aggregator using the proposed mechanism. (d) The total payment received by the aggregator using the flat price scheme. (e) Difference between the total payment to aggregator using the proposed mechanism and that using the flat price scheme.

optimal contracts that are shown to be good approximations to the theoretically optimal results.

\section{REFERENCES}

[1] "Electrification roadmap: Revolutionizing transportation and achieving energy security," Nov. 2009, [Online]. Available: http://www. electrificationcoalition.org/reports/EC-Roadmap-screen.pdf,

[2] W. Kempton and J. Tomic, "Vehicle-to-grid power implementation: From stabilizing the grid to supporting large-scale renewable energy," J. Power Sources, vol. 144, no. 1, pp. 280-294, Jun. 2005.

[3] W. Kempton and J. Tomic, "Vehicle-to-grid power fundamentals: Calculating capacity and net revenue," J. Power Sources, vol. 144, no. 1, pp. 268-279, 2005.

[4] C. Guille and G. Gross, "A conceptual framework for the vehicle- to-grid (V2G) implementation,” Energy Policy, vol. 37, no. 11, pp. 4379-4390, Nov. 2009.

[5] J. Xu and V. Wong, "An approximate dynamic programming approach for coordinated charging control at vehicle-to-grid aggregator," in Proc. IEEE Int. Conf. Smart Grid Commun., Oct. 2011.

[6] D. Wu, D. C. Aliprantis and L. Ying, "Load scheduling and dispatch for aggregators of plug-in electric vehicles," IEEE Trans. Smart Grid, vol. 3, no. 1, pp. 368-376, Mar. 2012.

[7] S. Han, S. Han and K. Sezaki, "Development of an optimal vehicle-togrid aggregator for frequency regulation," IEEE Trans. Smart Grid, vol. 1 , no. 1 , pp. $65-72$, Jun. 2010.

[8] E. Sortomme and M. A. El-Sharkawi, "Optimal scheduling of vehicle-togrid energy and ancillary services," IEEE Trans. Smart Grid, vol. 3, no. 1, pp. 351-359, Mar. 2012

[9] A. Ipakchi and F. Albuyeh, "Grid of the future," IEEE Power Energy Magazine, vol. 7, no. 2, pp. 52-62, Mar. 2009.

[10] C. Wu, H. Mohsenian-Rad and J. Huang, "Vehicle-to-aggregator interaction game," IEEE Trans. Smart Grid, vol. 3, no. 1, pp. 434-442, Mar. 2012.

[11] Z. Fan, "A distributed demand response algorithm and its application to PHEV charging in Smart Grids", to appear, IEEE Trans. Smart Grid.

[12] J. Laffont and D. Martimort, The Theory of Incentives: The PrincipalAgent Model, Princeton University Press, 2001.

[13] D. Fudenberg and J. Tirole, Game Theory, The MIT Press, 1991.

[14] S. Boyd, L. Vandenberghe, Convex Optimization, Cambridge Univ. Press, Cambridge, U.K., 2004.

[15] D. Kirk, Optimal Control Theory: An Introduction, Prentice-Hall, 1970.

[16] A. Seierstad, K. Sydsaeter, "Sufficient conditions in optimal control theory", International Economic Review, vol. 18, no. 2, pp. 367-391, June, 1977. 